\title{
ELECTION SYSTEM BASED ON BLOCKCHAIN TECHNOLOGY
}

\author{
Noor Mohammedali and Ali Al-Sherbaz \\ Department of Computing, Northampton University, Northampton, UK
}

\begin{abstract}
There is no uncertainty that the chroma currency is very popular for the transaction information stored in the block of blocks. To initiate the importance of blocking, there is only one distributed ledger in which data is stored, since many of the centers in the blockchain network limit enough records for these records. This document proposes a decision-making framework based on innovation to create a secure, unjustifiable, accurate and transparent framework for voter privacy. In addition, it is voted and declared in this context that the race has a short period of time, since it is automatically registered in the table. This framework also gives society confidence in its legislation when applying this technique. In this framework, an administrator can add a candidate and a voter to the block. In different hands, a voter can log into the framework and then decide in favor of a candidate that the details of the vote should be stored in the block. In addition, each square in the blockchain innovation that relates to the previous square contains the hash of the previous square, and each square contains explicit data based on the square footage. The hub is connected by a peer network in this framework, with all the hubs in the block site network that have a complete duplicate block.
\end{abstract}

\section{KEYWORDS}

Blockchain, Election System, Proof of Work, Smart Contract, E-voting, Peer-to-peer Networks, Bitcoin, Ethereum, Democracy.

\section{INTRODUCTION}

Since 2004, many nations have used the electronic democratic framework to vote and vote within a short space of time. This type of problem overcame many difficulties and attacks that could alter the story's impact. As this framework uses a database that stores all voting information that points to a disappointing purpose. Since the past, innovation has been used in decisions called "blockchain". For example, this innovation has a number of highlights, permanent and safe, that select the exact shape and are therefore used by the driving designers to develop a career framework. Moreover, the democratic result is a short time.

In October 2008, Satoshi Nakamoto created a block substance in Bitcoin. The electronic Peer-toPeer-Silver system used in 2009 the Proof of Work (POW) in cryptology [1] to execute in many applications death with the Bitcoin cryptocurrency. In 2013, Vitalik Buterin's Ethereum network was completed, and he was able to register the application that supports innovation [2]. Gavin Wood completed this material in 2014 with Ethereum Virtual Machine (EVM). Besides, Bitcoin and Ethereum talked about the step's innovation is taking for blondes [3].

Similarly, many applications have used block blocks to solve many problems. For example, a recovery replacement record that provides a voting frame that follows a single item and a personality. Regularly, nobody can change the information stored in a remote restricted network.

All of this data was used to calculate the SHA256 e-zine. All square interfaces with the previous square do not respect the past, respecting the history and the main square called Genesis Square. 
There are also three types of blockades, for example open, private and syndicated blockades [4].

Many electoral systems were proposed but rejected the fact that some vulnerabilities had a fixed number of voters and were of no value to the administration or associations. These systems used Bitcoin or Ethereum as an essential voting system to see the voting available on the block page they organized. Also, the marked ballot is marked with a mark (organizer and inspectors).

Occasionally, when the programmer gives out much more votes than the ordinary excavator that obstructs or attempts to invalidate it. Since the system has been processed by the ordinary and the programmer, despite the negative record previously placed on resources in his facilities Has.

Most applications received in one industry used the Ethereum wallet for the voting transaction and made the Ethereum wallet available to all voters. All purses used in elections have different currencies. On the contrary, the data of the voters are disseminated in an agreed database or a database like Couch base. As each of the centres in the system has been repeated, and the extra space available is expensive in block blocks. The use of open electoral camps can make it easier for individuals to build an electoral campaign on the electoral base and within the framework of electoral correspondence and the convocation base.

When we run our framework, all hubs in that system are linked, and duplicate tuning is placed in all hubs in the system. All voters were able to vote with their clever tools. First, our framework will review our personality in the block in the possibility of being transferred to decide in favour of a more competitive person. Second, our framework will act as a transaction in free space. From that moment on, the election approach could see the outcome of the vote by spending all decisions in favour of each competitor. Finally, the mob voting information stored at that time is collected and checked to obtain legitimate and invalid information for storing the essential information in the decentralized blockchain database. The results of this test are of paramount importance to protect the election results from misrepresentation.

At this level, cell innovation can work and enable the secure and non-negative transmission of basic data practices and security. The problem today, however, is the adaptability of the blocked site: as DLT gradually detects things on the Internet. It probably leads to two IPOs, and we understand that this is the reason why it is not. The power arises. This innovation, For example, Bitcoin 7 TPS, Litecoin 56, Ethereum 25, BCH 61 and Tron 2000, can all be improved. A superposition of rules, such as Network Lightning in an RTB or LTC provides the flexibility that sources cannot manage.

The remainder of this document is organized as follows. Section 2 examines the crafting of cellular innovation in election campaigns. Section 3, Our Commitment and the Framework Engineering Model. Section 4, Results and Evaluation of the Framework. Finally, the article ends in section 5 .

\section{BLOCKCHAIN}

At this level, blockchain technology could work, allowing normal and natural security to transmit information and installation exercises in a safe and non-detrimental manner. The problem now is, in any case, the flexibility of the blocking position: if the DLT is more related to Internet things. It probably survives a large number of IPOs per second, and we understand that this is the purpose without it. The power comes out. This technology. For example, Bitcoin 7 TPS, Litecoin 56, Ethereum 25, BCH 61 and Tron 2000 can be amplified. The overlay of a principle, like Network Lightning in BTC or LTC, is ready to provide adaptability that sources cannot afford. 


\section{Motivation AND RELATED WORK}

\subsection{A website that Builds E-voting System}

In a decentralized setting, there is no disappointment. In this way, a programmer can not attack the frame even if it attacks a single centre in the frame that can not affect or attack the frame. Stream My Vote is a US-used product that is used for a trusted trust voting framework that relies on restriction innovations to continuously track the voting process. Problem You limit the result and show no particular end. Election Runner is another electoral framework in which the innovation of blondes is used. This framework uses the different workspaces of the client for Android, iOS and website. Two hundred fifty-six pieces of hashed encryption are used in this framework to secure them.

Nevertheless, it may be possible to import voter information from the CSV, a spreadsheet record, rather than moving forward continuously. If each voter creates an ID, the voter uses it only once. The point of no power in this framework can still be used in school, university or society, but not for the benefit of the legislature. Likewise, you can understand who chooses to favour this choice by name. Understand your identification. Besides, the selection will be left to audited endorsers and therefore, must have 100\% confidence in Electronic Runner and an on-site expert [6]. It uses a European company called DCent, which uses free coin on a block-block basis using a device with a cryptographic capacity [7]. On the other hand, Secure uses an Australian company called VoteFlux. Vote [8]

\subsection{Academic Research Paper}

The Confederation of the Russian Federation (NSD) announced a joint venture that would give bondholders an electronic voting model. The block-based e-proxy voting model records the policies directly in the distributed registry, which uses every member of the chain immediately and without delay said by Sergei Putyatinski. IT Director at NSD, saying his framework 80 will come could be transactions per second, and I want to create the framework for 300 transactions per second [9]. Previously, Hyperledger teamed up with NSD to provide an electronic voting framework for intermediaries, who are at the top of the NXT fork, earning 80 more votes for each, which translates into 160 transactions per second. Similarly, they used block blocks according to the promised test to process the adaptability to handle up to 800 transactions per second [10].

In [11], they analyze the current framework and determine the size and load of each framework. As they do so, they recommend their framework depending on the block positioning technology. To make it a secure, persistent, and mysterious framework, as it has been used on the Web. It is assumed that the customer's device is secure and the customer once a ballot can lead. The result is permanent.

In this case, nobody can make the frame smaller. Also, there are a number of obstacles in its framework. Most likely, a hacker will provide a client technician with mobile programs that he can use to change the vote.

In [12], they used the visually impaired brand to defend and destroy voter decisions, and there is no compelling reason to reject them. In addition, they used an open block Web site to open it and used a similar information structure. That structure Bitcoin used to create an electronic voting framework without the stranger needing it. There are many shortcomings. The first, in the transmission of information, your assembly could reveal the IP addresses of the voters. After that, these ballots are amazing when sent to the blocks they organize. In addition, they provide two 
answers to this problem: obtaining admission to the block or encouraging voters to encrypt their ballot with an open key given by the coordinator. Third, the disclaimer occurred at some point when the organizer and the inspectors proposed making a substantive and invalid vote with their brands. On the other hand, there are some security issues: at irregular intervals, they can not find communication between voters and ballots unless the second key is open. The moment the voices are not misunderstood, the network will break the mark. Finally, a retransmission attack with the legitimate peer network (P2P) will not work, and voices will be checked as soon as the malicious centres start at a different time.

In [13], they clarified how to block bloc works after polling, what procedure is used for deciding how that message denied the use of the electronic SHA256 site and which was shelved in a square. After verifying approval of the box and each box's interface with the previous box using the previous hash key and timestamp. Gather information from the ticket stored in an open database that customers need so nobody can change that information. In this case, the democratic result is not ignored. To break down and test the result, they used the Python programming language PyCharms software to store the procedure of each square in the tree in 0.24 seconds. And the normal ability to store that information in 216.04 bytes for each square to process.

In [14], they suggested the advantage of using a P2P network with block technology to achieve effective resuscitation when the driver sends the tire to the receiver. In this case, who knows the beneficiary but does not know it. The driver of all city centres. Consequently, they proposed a proprietary technique for approval and rating systems. They also presented the feasibility and quality of this component. There are many difficulties with the P2P application, eg. For example, a versatile network connection to the network where the number of clients on the network is unknown and which are the following, and there is no default trust between them. In the P2P barrier implementation, the centre of the freeway centre offers another exchange centre.

The central securities depository in the Russian Federation (NSD) announced a pilot project to provide bondholders with an electronic reconciliation protocol. The e-proxy voting prototype records the success between the sender and the recipient. Subsequently, a confirmation for secure verification by mutual encryption is sent to the mine lock. It comes from a selfish user.

In [15], they proposed an electoral system that uses Blockchan's private network based on the Ethereum API and allows voters to update their votes during the election period. The advantages and disadvantages of this technology lie in the electronic voting system. This electoral system offered many possibilities, such as transparency, information integrity, protection, accuracy, enforcement and accessibility. Each node has a complete duplicate in its distribution network. There is no single specialist, and his users remain unknown in a dial-up network.

In [16], they recommended that the electronic voting blocks enable electronic voting (BEV) using digital currency in your voting system by creating a bag for the voter and the candidate. Every voter has a medal. This single base is used once to vote for a single candidate when the vote cast from that single base has been transferred to the candidate of choice of the voter. In addition, the system allowed voters to change their vote/update before the deadline, and voting must be anonymous over the network. They also recommend many examples of the voting system that uses block blocks, for example, Voatz. For example, in addition to the secure, consistent, accurate and intelligent monetary system, voters can vote remotely. In addition, they proposed the challenge facing the voting system before blocking blocks, and after blocking blocks, for example, the Blockchain system required a lot of energy to perform authentication and approval. 


\subsection{Countries used Blockchain for Voting}

On January 10, 2016, an organization called the APLA was formed using blockchain recording technology as a cornerstone to protect and exploit the democratic functioning of governments such as the United Arab Emirates, India, Russia and the West Indies. Netherlands or organizations in Dubai. These application environments operate in a gradual framework for admission to information, interfaces, and grand contracts [17], [18].

In Argentina, there is an association called Democracy Red and DemocracyOS is a free and opensource online software that is used for democratic purposes. There are three levels: Collect political questions, talk about these topics, and decide on an explicit proposal. The goal of this task is to create a democratic stage for every city or government. In addition, Mexico has taken this step to gather criticism of people under the Open Source Information Agreement, and an association in Tunisia called I Watch used this open-source tool to give people a more fundamental voice when they do vote [19].

In India, the company is trying to exploit the obstacles in its business. Andhra Pradesh has for some time been the first block-block technology state to register its records, computer-aided identity, cross-border payments and transportation as test companies. These use cases for pigs depend on conceptual evidence. They also send a group to numerous conferences or workshops to understand the technology and its implications for their business. And also, to face a number of challenges in using blockages, vendor/phase, onboard partners, development environment, security issues, and Cohesion problems [20].

In Boston, USA UU., January 8, 2018, a blond company and an IBM person named Voatz who use blocking technology to make transparent, efficient and secure agreements. In addition, they focused on four key regions to achieve success, such as Versatile security, voter darkness, unsurpassed nature and clarity through a secure mobile phone, multi-source identification assurance process, and biometric data Motivation and assurance are based on Blockchain. In this context, the declaration of identity takes twenty to four hours and strengthens one million votes per second. It is also used in government, organization and university elections in private or open elections [21] [22].

In the New York Times, Washington Post, there were shortcomings in 2016 in the presidential race. In the US elections of November 2018, Voatz was therefore used as a portable application of her choice to cast unreliable votes and use blocking technology on a permanent basis [23].

In Australia, Horizon State has a defence system based on blocking technology. Of course, there are plenty of locations for your items: a secure structure, a warning of problems, voters can access the material to the crusher, and investigative tools tell you about the viability of individual fights and how did the voters behave.

In December 2017, Russia had a program called Active Citizens Program. This program uses a private phase of Ethereum to improve voting and strengthen trust between the voter and its legislator. In addition, 1000 transactions per minute will be settled if the entire democratic voting result is recorded in the registers and all the centres that can see the result [25] [26]. The Indonesian government used a portal as a portable application of their choice on the island of Sumatra in July, which was created by the Australian organization Horizon State. They want to grow as a nation beyond their wealth [27]. 


\section{EVAlUATING BLOCKCHAIN AS A SERVICE FOR E-VOTING}

For implementing and deploying our smart election contracts; the table below shows the comparison between three different blockchain structures. These are Exonum, Quorum and Geth.

Table1. Framework Evaluation

\begin{tabular}{|c|c|c|c|}
\hline & Exonum & Querum & G0-Ethereum \\
\hline Consensus & Custom-built $\mathrm{BFI}$ algorithm & QuorumChain, IBFT and Ratt-based consensus & PoW, POS and PoA \\
\hline Transactions $\mathrm{p} / \mathrm{s}$ & up to 5000 transactions $\mathrm{p} / \mathrm{s}$ & Dozens to hundreds & Depends \\
\hline Private support & Yes & Yes & Yes \\
\hline Smart Contract Language & Rust & Solidity & Solidity \\
\hline Pıvgramming Language & Rust & Gu, C, JavaSciipt & Gu, C, Javascripl \\
\hline Decentualizeal & Yes & Patially & Optional \\
\hline
\end{tabular}

\subsection{Exonum}

The exonum Blockchain begins to complete its complete execution with the banner programming language. Exonum works for blocked private pages. Byzant calculation is optimized to reach the system. When calculating this calculation, Exonum can support up to 5,000 exchanges every two days. In summary, the blockade is that Rust is the main programming language in the current process, which limits designers to developments that can be accessed in that language. To overcome this limitation, Exonum tries to present Java connections and an autonomous representation of the staging interface so that Exonum becomes friendlier with engineers sooner rather than later.

\subsection{Quorum}

Is the Convention based on records of provision of funds in Ethereum with Exchange / Contractual Security and recently agreed systems? Geth is a fork and will be renewed after Geth's escape. The majority of the instrument was changed by the majority and continued to reflect the calculations of a union-based agreement. By using this agreement, it will be possible to help every second of the bridges exchange hundreds.

\subsection{Geth}

A Go-Ethereum or Geth is one of the three original uses of the Ethereum Convention, and cleverly executes custom contract applications without personal time, restriction, blackmail or external barriers. This structure forms the basis for improvements throughout the Geth Convention and is the most technically mature and most willing system. The second conversion (exchange rate) depends on whether the blocking is done as an open or private system. Because of these skills, Geth was the structure for which we put together our work. Any comparable structure comparable to recognized Geth capabilities should be considered for such frameworks. Protection Scenario from Relevant Attacks.

There are two types of public blocking sites called "Less Authorization" that indicate that anyone can enter the system and that private blocks called "Approval Blocking Blocks" indicate that the blocks are in an organization. Partnership when an approved customer can access these blocks [28]. Certainly, a cyber-security attack is gradually being extended to capture individual data or money information, especially in the case of barriers, there is a cyber-risk challenge, such as a computer-aided nature. In this way, cyber protection since blocking has been improved because it is secure, unchanged, simple and without any disappointing purpose and information encryption. The private blocking connection must protect the system from attacks as long as the systemic 
attacker can access the information. Therefore, the company must fully encrypt the blocking information to ensure that no illegal capture can be performed. Information without authorization [29].

Distribution accounting technologies (e.g., the Bitcoin settlement system Ethereum and Hyper) used agreed calculations according to their currency type. The DTLs had numerous security challenges, e.g. Denial of malware denial service and mining). The cases are:

1. Cases of Transaction: digital signature is used in each form of transaction. This transaction is transferred between the centres for validation. In this case, the attacker can delay or reject the transaction.

2. Spam attacks: In this case, the entire system is cut off.

3. Malicious Contract: In this case, the attacker creates a contract without an Ether. The moment the return capacity runs long enough without determining the gas limit, the application will continue until the maximum call is reached.

4. Anonymity: Become familiar with the address of the customer who has informed the attackers about the history of the customers and their transactions in the system.

5. Mining Group: Control the system by getting half the power of the hash. Use the Trojan frame to take control of the computer and use timestamps to speed mining.

6. Opportunity Attacks: A wrong fan was transferred in the system after the system mates had changed by adding new friends to the system without affecting the authentic centres.

7. DDoS (Distribution Service Rejection Attack): Lots of information floods into the system without reaching it for transactions [30].

For authorization interconnections and purposes between barriers, the elliptic curve algorithm (ECDSA) was used for the blockchain application. This algorithm depends on secp256k111. In addition, this type of algorithm used a signature in digital form in several steps. First, the information that uses this information is scanned with the private endorsement key. Therefore, the signature checks the signature using the Subscriber's opening key and the corrupted information to re-sign the signature recovered [31].

The SHA512's cryptographic algorithms used an information-sharing framework because they spoke with a secure algorithm that could be accessed today. In addition, there are different type of alternative attacks, such as prepaid attacks, impact attacks and crash simulation. In the light of Moore's Law, the difficulty of the square will increase after a while, and there will be two regular checks. In this way, the malicious attack could trigger the chain attack on T1 if T2> T1 [32].

Therefore, there are many cases of piracy that have differed from one another to protect our system from attacks.

\section{Scenario 1:}

Q: What happens if the blocking framework has more than one administrator?

A: If the blockchain framework has more than one administrator or one coordinator and consultant. They can participate with their keys to declare the invalid vote as legitimate and not useful for the decision-making framework.

\section{Scenario 2:}

Q: Why does the voting system not allow administrator to extract and recognize the exchange in the blocking system?

A: If the administrator can usually allow the exchange, an administrator can reject multiple votes that result from a voter for a particular claimant. 


\section{Scenario 3:}

Q: What happens if the voter performs a vote twice in the Blockchain system?

A: In the case of a double vote, the approval of the chain is wrong. In addition to these lines, the Blockchain is used twice against a ballot and not added to the block.

\section{Scenario 4:}

Q: What happens if a wrong registration enters the voting system and votes? Are these points correct or not?

A: An incorrect registration cannot be registered in the system because every character is verified with the information. The likelihood of this happening, however, is abused by the marking system for this vote. This vote will be rejected, and this vote will not be the same.

\section{Scenario 5:}

Q: What happens if the decision maker breaks down the decision-making framework, he creates in one way or another and downloads the complete duplicate data? Does the attacker have the opportunity to liberalize this data?

A: The attacker cannot recover the data because the attacker is almost certain to get the private key for the voter or administrator because these keys are created and canceled on their devices. In addition, the SHA512 calculates secure sites and does not balance them until then, as it requires a lot of processing power and takes a long time to recover data from a hash.

\section{Scenario 6:}

Q: What happens if voters include malicious software in their widget? Does this framework allow another program to use your information?

A: No, with this framework, no software can retrieve your information, read or write information from another application.

Towards the end, our framework has a number of security highlights, for example:

1- Link Restoration Structure: Blockchain was essentially a square of squares, using the square of the previous square from the square of the previous square, because this structure is in the block of blocks in the field. There is no difference in a square that you need to recreate to substantially implement this enhancement.

2- Privacy: Only the President can add voter and competition details to the frame placed in the block and add it to the chain block after dealing with the administrator's private key. Check this flag by opening the administrator password. so, nobody can retrieve this information. The electronic location calculation SHA512 is used to cool the square information even if it requires $1 \mathrm{MB}$ per hash. However, since the regular intervals are two arithmetic increments according to Moore's Law. To use the high mark, a protection calculation called ECDSA is used to sign the exchange on the square.

3- Avoid round votes: A voter can vote if he uses his private key to sign the exchange at that time. He announces the result. In this case, you will be prompted to create two flags of a similar voter when this is done, the approval toy is wrong, and this square will not be added to the block. 
4- Incorrect Character: The wrong registry cannot be used to log in to the framework because all voters enter the framework before the decision is opened. At the time the voter attains a legitimate personality, the voter can register and vote in favor of a candidate who needs a voter. When the voter votes, the vote is fair and added to the block.

5- Validation: Before a square is verified with the block chain, the approval of the chain and the approval of the current square and the previous square are verified. In the possibility that the approval is true, the square is added to the block.

6- Decentralized Planning and Survey: The voting result is questionable and will be considered for each centre in the block locations. The system design for this framework is distributed to a system.

All that is expected is that it is not self-evident that aggressive access to block data can recover, anneal, or read that data. It is because the data needs to be recovered by private key attacker. In general, these data are not enough for an attacker. Therefore, a cryptographic calculation is used to create a key pair that marks the data.

\section{SYSTEM ARCHITECTURE DESIGN}

The frame configuration is described in Figure 1, this structure becomes more effective and stronger. The below scheme contains three types of strings instead of three types of blocks in a string. The eldest has a first one, the voter has a second and the vote for the third. The current block for each chain associated with the previous block using the previous hash block. Similarly, each block in each chain must contain information, depending on the information structure with which it has its chain.

- The address of a sender, the address of a collector in a voting repository, or the disability of a vote, as indicated in the table, should be shown in Figure 1. These addresses were displayed as the opening key for these addresses. The name of the selected applicant is that of the transactions. Finally, this information is flagged before it is added to the reconciliation transaction.

- A wallet: A wallet has been created and this wallet is used to create a private and open key pair.

- An administration needs to add voter data to the voting system after checking its private key with the parent pair it is creating to confirm that the information provided by the administrator takes into account the square choices.

- A current hash of each square depends on the information structure of this square, with the aim that the substance of the square axis of the voter is not very similar to the applicant's square and does not represent the democratic square, as shown in the previous graph.

- All fields are joined to a previous field using a previous hash for the previous field. This makes this system permanent in the system, based on the fact that every adjustment in the entire previous field is made for each adaptation in a square information.

- SHA512 arcade algorithm for cooling the information. This algorithm is likely to delay the system now and not use it without counting the client, but in two years the computer's performance will increase and this algorithm will protect the system from attacks.

- This system has an administrator who adds the data to the system and verifies the democratic outcome. 
- Management can see the result, but still cannot change it. If the administrator has to vote, you also need to sign up as a voter to vote for a candidate.

- The entire node in the system can always see the democratic outcome after voters voted for a more competitive person, and increase the democratic outcome when others vote.

- All system nodes are used to use a point-to-point network (P2P). All clients on the network have a complete duplicate of the block. If the new square has been added to the network in these lines, that square will be in each of the nodes in its chain.

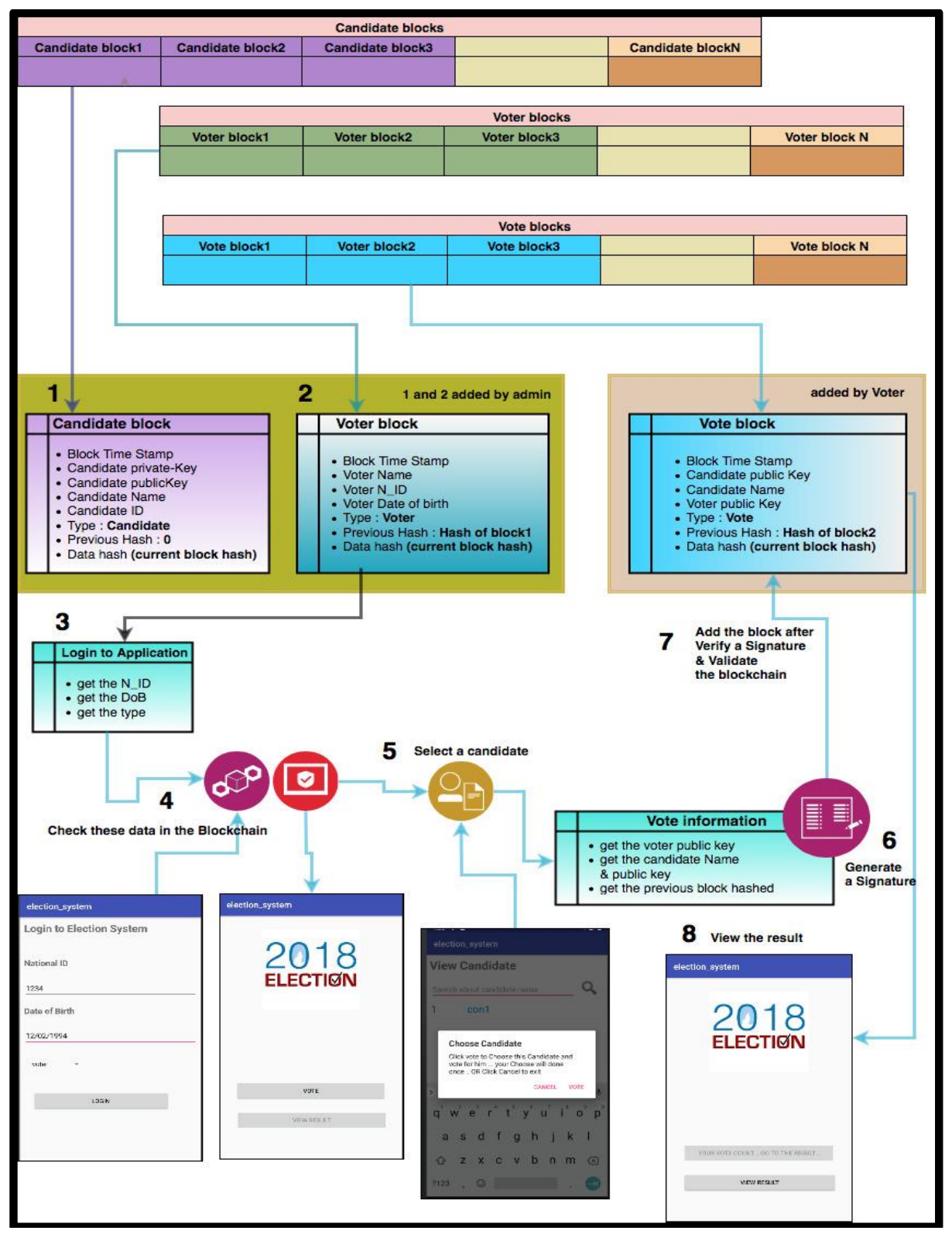

Figure 1. Three chins each one has one type of block

In the chain of a candidate, the chain stores each of the blocks identified by the candidate and taken into account by the administrator. In addition, these data must come from the candidate lock file for the administrator and voter to view. In a dial string, the chain stores each of the blocks identified by the voter data and taken into account by the administrator. In addition, this 
information is visible to the administrator after being added to the dial string. The moment the voter logs in using his information in the frame, the voter with national identification, his date of birth and his type checks the information at the voter block's location on the occasion he has not given a chance to himself to register in the voting framework.

This framework also creates a pocket for every voter, this voter has a central pair for that voter and is used by the voter when a candidate votes on a candidate. In these lines, when the voter chooses a candidate to vote, he receives the details of the candidate he has received from a candidate for a block, a voter's opening key, and an earlier block signature hash, depending on the pair's voter generator from key to the reconciliation process. At this point, when denied, verified and validated by the democratic blockade, this information blocks information such as timestamp, type, previous hash block and current hash block. In addition, the Democratic chain maintains all the blocks that contain voting information. The voter enters this data along with the chain after selecting a candidate for voting in exactly the same way. The democratic result is taken from the democratic chain and submitted to the administrator and the voter to determine the number of votes for each candidate.

For all purposes, the SHA512 extension algorithm is used to redeem the block information. He used the same thing that was used and spoken earlier. In addition, the digital signature algorithm of the Elliptic Digital Signature Algorithm (ECDSA) was used to verify, verify and validate the character. Before adding to the block. If the information attached to the chains is not changed on the basis that each setting in the block must be changed and saved for each pasture of the last block. The entire hub in the system is correlated using a peer-to-peer (P2P) arrangement. Given the number in the table in Figure 1:

1) A candidate squad comprises a candidate squad. In addition, the general square data examined above includes private and open keys, applicant name and ID.

2) The administrator also adds the voter list. In addition, the general data includes the fields for identifying the voter and the date of birth.

3) When the administrator inserts a voter into the block, the voter can log into the frame with his national ID, birthdate and type.

4) Probably the wrong record will not capture the decision framework. The administrator adds all real information with a real personality to the frame. In this way, the character in the frame at this point is likely to indicate that due to the nature of the voter, he provides the voter with a key pair to vote with a competitor.

5) When the voter starts, the applicant will receive the opening code, the name and the opening code of the aspiring person. Thereafter, a tag is used that uses the voter's private key to sign the transaction at that time. You declare this transaction with your opening key. Toward the end, when checking the approval of the chain, if it is serious, this square is added to the block position and the voter is placed at that point to see the result.

6) The ECDSA expressed as a protected calculation is used with the highest signed and confirmed highest grade after the square has been added to the decision.

7) The open-key voters were included in the bloc after the voter voted for the candidate. This vote also takes place once for each voter.

8) Once that voter has cast his vote as soon as that framework makes him democratic again, when the voter closes the meeting and re-signs each time the frame unveils his voice. In this aspect, this voter can see the result. 


\section{SYSTEM RESULT AND EVALUATION}

In this the user has to put the username and password so that admin can login to the home activity. To add voter and admin; the home activity belongs to the privilege of the admin.

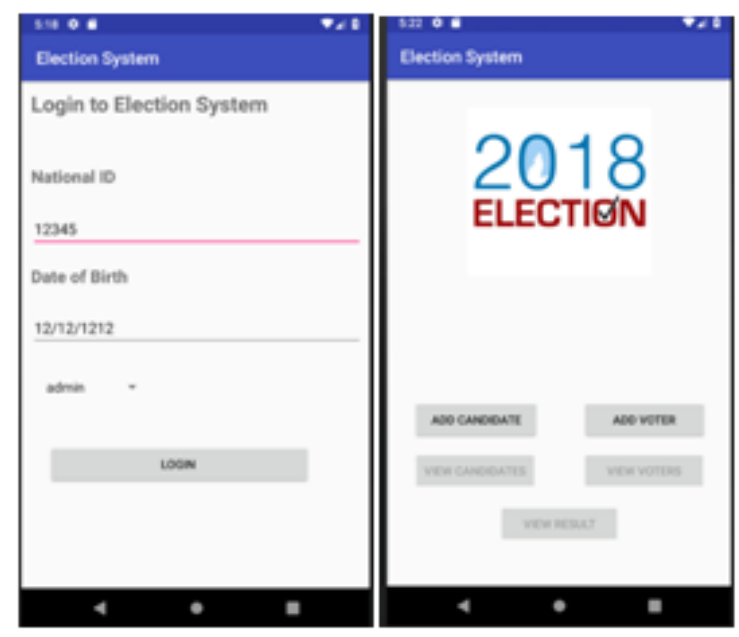

Figure 2. Login and Home activity

Add a voter or candidate to the system as in the screenshot below.

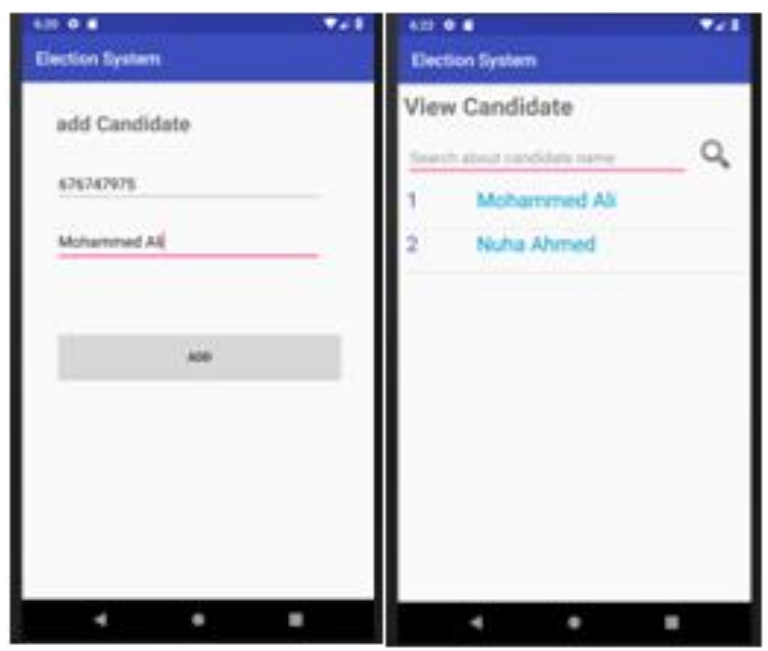

Figure 3. Add and View the Candidate

The main one inhibits that "the Geneses block" does not have an incentive for the previous hash. The moment the square is added to the fabric later, you will find the previous hash for the main square. 
International Journal of Computer Science \& Information Technology (IJCSIT) Vol 11, No 5, October 2019

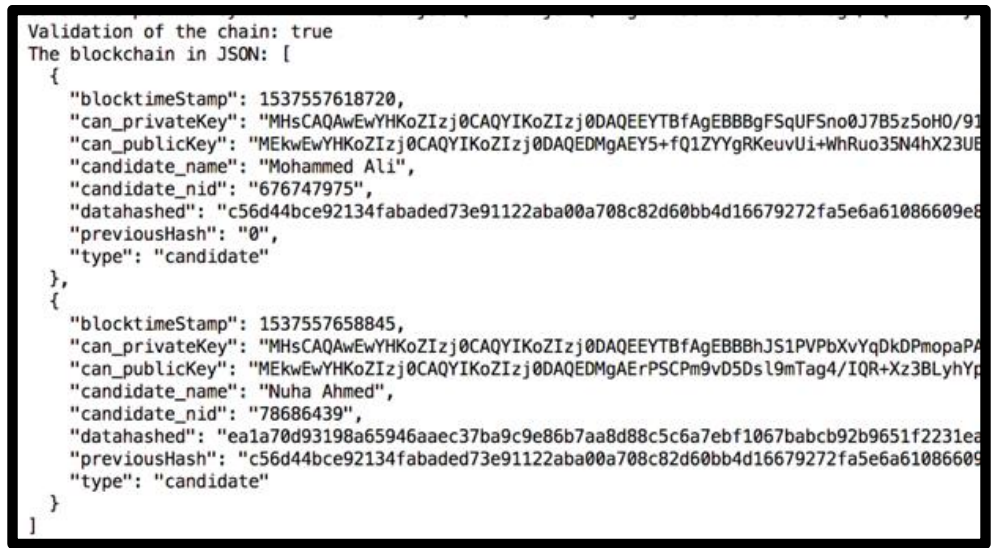

Figure 4. Save the candidate data in Blockchain

As the screen shot below shows that in system the Admin will add a voter.

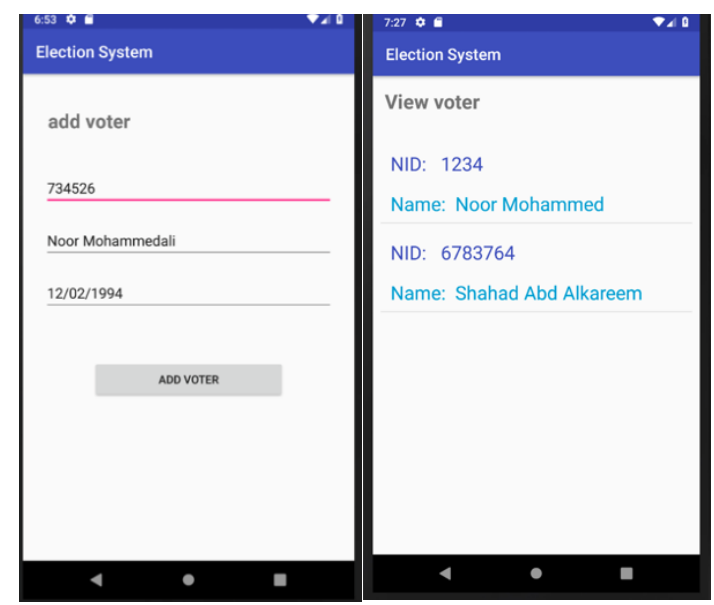

Figure 5. Add and View voter

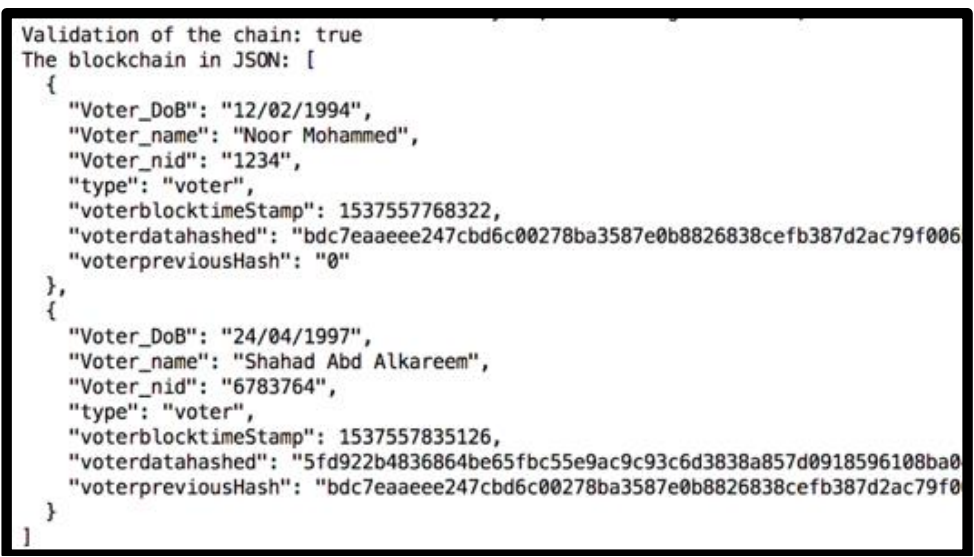

Figure 6. Save the voter data in voter chain

From that moment on, the voter registers with his national identity and his date of birth in the context. Our framework examines the personality of the voter, if it is legitimate to send him to the Chamber to vote. In addition, the result may be imperceptible until voters vote for a nominee added by the administrator. 


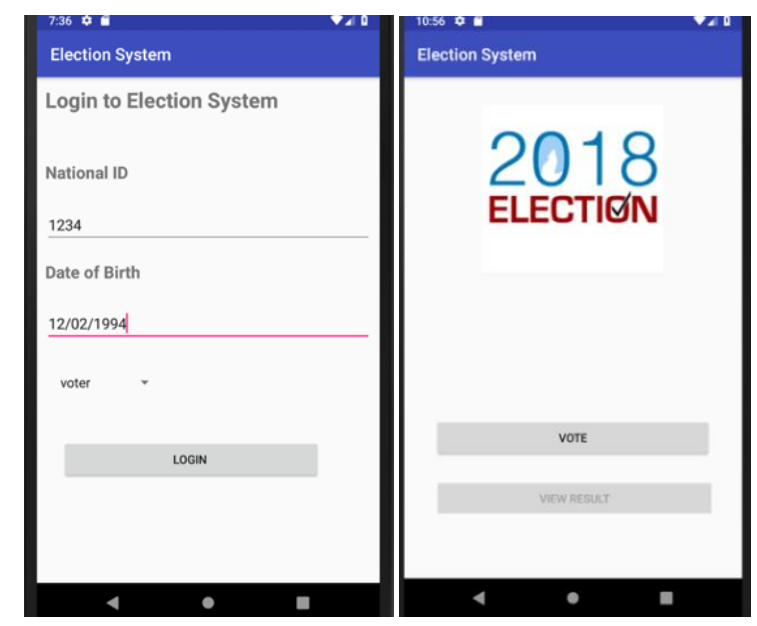

Figure 7. Voter login to the system

At the time the voter receives a vote to vote, a ballot should be considered a competitor, as shown in the first screenshot below, at the time the voter clicks and participates in the soft speech. Enable the voter to vote or release the decision and return to action at home, as shown in Figure 8.

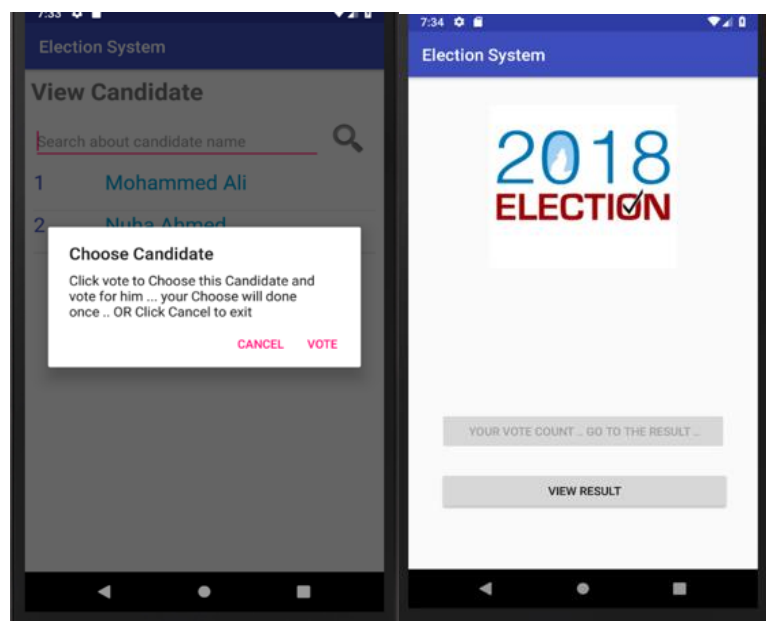

Figure 8 . Voter vote once for a candidate

At the point where the voter smoothly clicks on holding the poll in the discourse, the voter is open, and the name of the applicant and his opening key confirm and approve the democratic place after signing the information. 


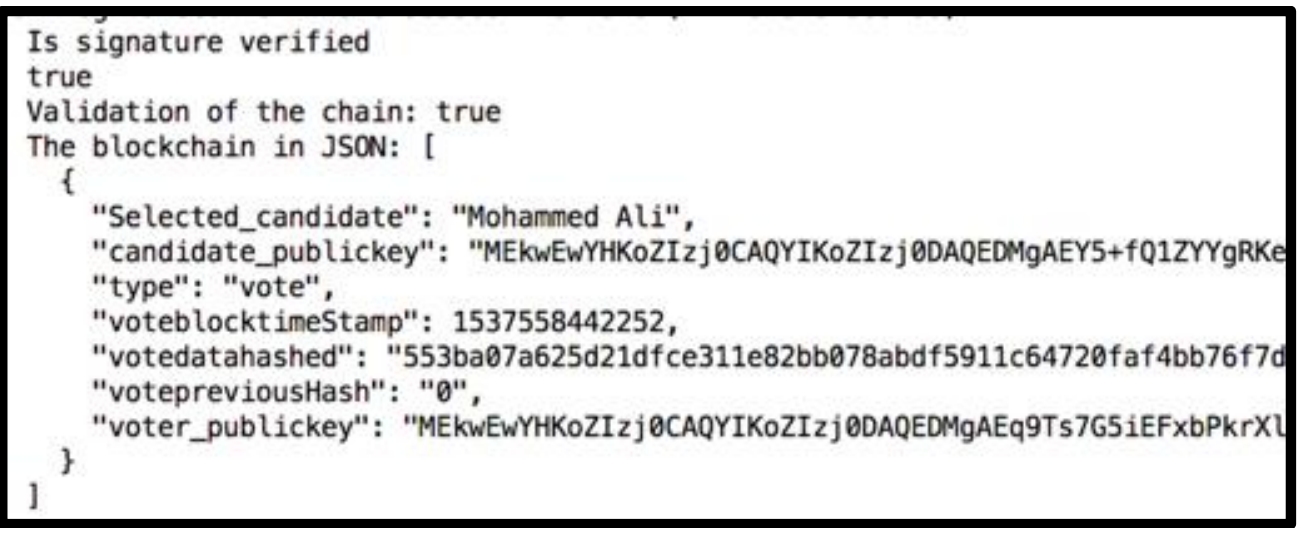

Figure 9. Save the vote date in vote chain.

Presently the voter and the administrator can see the vote result, as in the screen capture beneath.

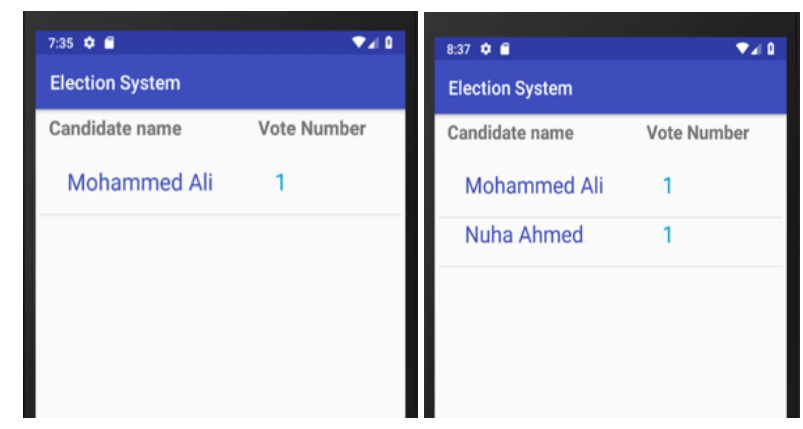

Figure 10. View the voting result

At the point when the second square of vote added to the framework will include the democratic chain as in the screen capture beneath.

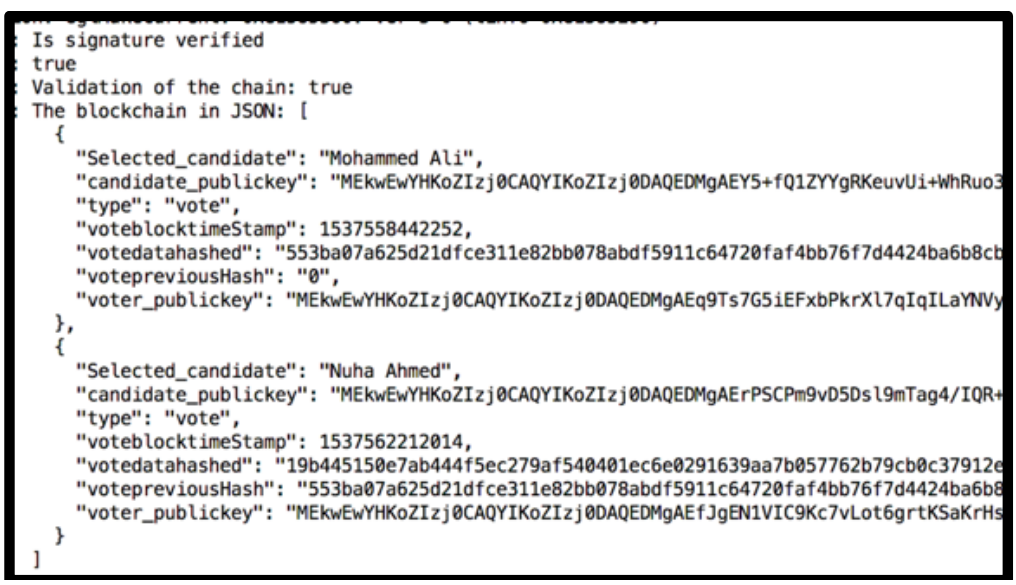

Figure 11. Save the second vote to vote blockchain

Another form of design is shown below: 


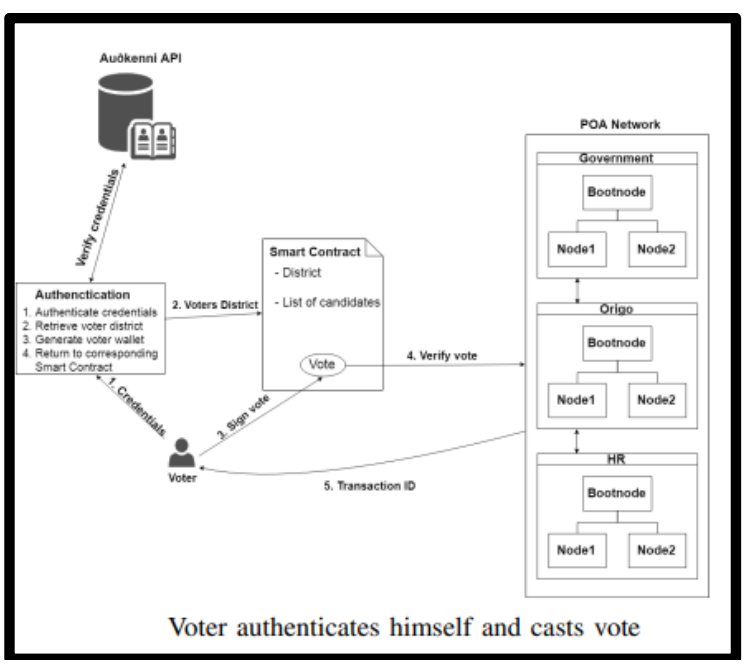

Figure 12. Vote authenticates himself and casts vote

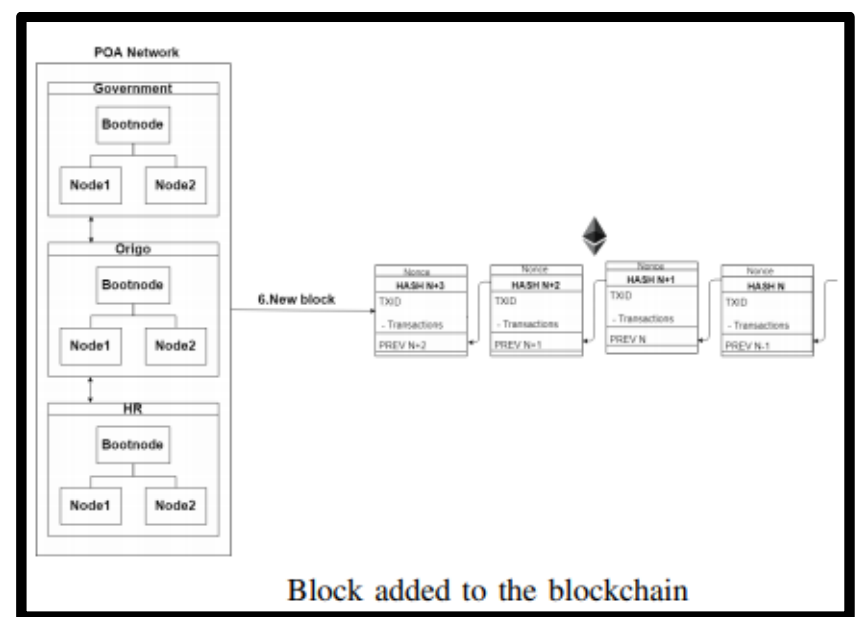

Figure 13. Block added to the Blockchain

1) Any qualified voter may use any computer in any democratic area to conduct a vote, since the ballot contains information that the voter must pass from the pocket to the comparator. For a customer to be verified effectively, a significant ID and PIN number must be displayed in a democratic location using a Nexus card user and programs.

2) If the declaration is effective, the ideal contract in relation to the decision is preferred. The results for the aforementioned race are weak and many of the people who come are coming.

3) When a voter has selected a competitor and makes their own decision, the voter continues with his signature by returning the PIN number associated with his identification.

4) After voting for the voter, the related center continues to verify the voting information in which the voters are working with the contract. Given the possibility that the aforementioned regional configuration proposal confirms the voting information, the voting information must be compared with the range center that is making the comparison.

5) If most of the local centers approve the voting information according to the specific vote. The customer will receive the replacement ID at this time to compare the exchange of votes as a QR code and print the replacement identification option. At the time of voting and review, a 
vote in the contract is in accordance with a vote of the Assembly that was elected for the vote. This benefit is used in terms of a contractual structure to decide on each of the democratic areas. The main character is a visual representation of the methods we have simply explained.

6) All transactions captured and confirmed in continuous square time are executed in the block once the square time has reached the time limit shown in the figure. With each new square added to the location of the block, each district center fills the register.

\section{CONCLUSION}

The blockchains are decentralized. As per these lines, there is no single purpose behind disappointment. What's more, there is no compelling reason to pick up the trust of the outside individual. The motivation behind this record is a safe and steadfast electronic democratic framework. The whole hub, which is associated during the decision and the democratic outcome, is checked effectively and considered by the voter. Numerous objectives have been set to build up this framework. Essential research was directed to comprehend the expansiveness of issues and some shortcoming was found in momentum applications. These endeavours have been considered as applications to take care of these issues. Fundamentally a Java programming language with which the framework was created when organizing a portable application so as to encourage the decision in favor of the voter. Then again, it is less expensive, more vitality proficient and more secure. What's more, the framework has been executed, for instance, the social affair of the signup development, the consideration of the applicant and the voter, and the perception and representation of the democratic outcome. The SHA512 site rather than SHA256 has utilized it to expand security in the event that you use it later on. Likewise, the quadratic competitors put from applicant 1 to $\mathrm{N}$ in the chain of up-and-comers obstruct a voter from a voter 1 to $\mathrm{N}$ in the chain of voters. Towards the end, the democratic box was added to tune 1 to N. For future improvement, this framework will be utilized in different strides, with framework data put away in a cloud or conveyance database.

\section{REFERENCE}

[1] S. Nakamoto, "Bitcoin: A Peer-to-Peer Electronic Cash System," 2008. [Online]. Available: https://bitcoin.org/bitcoin.pdf. [Accessed 21 February 2018].

[2] V. Buterin, "Ethereum: A Next-Generation Generalized Smart Contract and Decentralized Application Platform," 28 December 2013. [Online]. Available: https://blockchainpapers.org/items/show/2. [Accessed 15 March 2018].

[3] G. Wood, "ETHEREUM: A SECURE DECENTRALISED GENERALISED TRANSACTION LEDGER,” 2014. [Online]. Available: http://gavwood.com/paper.pdf. [Accessed 15 March 2018].

[4] R. Richardson, "Block chain startups signal new approaches to data integrity," 2015. [Online]. Available: https://searchsecurity.techtarget.com/opinion/Block-chain-startups-signal-newapproaches-to-data-integrity. [Accessed 10 May 2018].

[5] F. M. Vote, “The Online Voting Platform of The Future - Follow My Vote," [Online]. Available: https://followmyvote.com/. [Accessed 15 February 2018].

[6] E. Runner, "Build a Secure Online Election for Free | Election Runner," 2017. [Online]. Available: https://electionrunner.com/. [Accessed 30 May 2018].

[7] Dcentproject.eu, “Dcent,” [Online]. Available: https://dcentproject.eu/. [Accessed 31 May 2018]. 
[8] Securevote, "Secure.vote," 2018. [Online]. Available: https://secure.vote/. [Accessed 31 May 2018].

[9] P. Rizzo, "Russia's Sole Central Securities Depository Trials Blockchain Voting," 24 May 2016. [Online]. Available: https://www.coindesk.com/russia-national-settlement-depository-blockchainvoting/. [Accessed 15 May 2018].

[10] G. Prisco, "Russia’s National Settlement Depository Successfully Tests Blockchain-Based E-Voting System," 24 May 2016. [Online]. Available: https://bitcoinmagazine.com/articles/russia-s-nationalsettlement-depository-successfully-tests-blockchain-based-e-voting-system-1464198071/. [Accessed 15 May 2018].

[11] A. B. Ayed, "A Conceptual Secure Blockchain- Based Electronic Voting System," International Journal of Network Security \& Its App lications (IJNSA), vol. 9, no. 3, 2017.

[12] Q. W. Yi Liu, 2017. [Online]. Available: https://pdfs.semanticscholar.org/5b6a/0b0ff2c574d9bb8bad9e191b22f44c92add7.pdf. [Accessed 25 May 2018].

[13] B. R. Rifa Hanifatunnisa, "Blockchain based e-voting recording system design," Telecommunication Systems Services and Applications (TSSA), 2017.

[14] H. L. X. C. Y. L. C. Y. L. S. Yunhua He, "A Blockchain Based Truthful Incentive Mechanism for Distributed P2P Applications,” IEEE Access, vol. 6, pp. 27324 - 27335, 02 April 2018.

[15] A. G. R. N. A. K. M. Freya Sheer Hardwick, “ E-Voting with Blockchain: An E-Voting Protocol with Decentralisation and Voter Privacy," 3 July 2018. [Online]. Available: https://arxiv.org/abs/1805.10258. [Accessed 23 August 2018].

[16] J. V. Nir Kshetri, “Blockchain-Enabled E-Voting,” IEEE Software, vol. 35, no. 4, pp. 95-99, 2018.

[17] apla, “apla," 2016. [Online]. Available: https://www.crunchbase.com/organization/apla\#sectionoverview. [Accessed 15 August 2018].

[18] egaas., "what-is-Apla," 2016. [Online]. Available: https://egaasen.readthedocs.io/en/latest/introduction/what-is-Apla.html. [Accessed 15 August 2018].

[19] D. e. Red, "DemocracyOS," 2017. [Online]. Available: http://democracyos.org/. [Accessed 15 August 2018].

[20] Deloitte, "Blockchain technology in India Opportunities and challenges," April 2017. [Online]. Available: $\quad$ https://www2.deloitte.com/content/dam/Deloitte/in/Documents/strategy/in-strategyinnovation-blockchain-technology-india-opportunities-challenges-noexp.pdf. [Accessed 25 July 2018].

[21] I. Voatz, "voatz,” 2018. [Online]. Available: https://voatz.com/. [Accessed 14 August 2018].

[22] P. N. A. LLC, "Voatz Raises \$2.2 Million Seed Round Led by Medici Ventures," 2018. [Online]. Available: https://www.prnewswire.com/news-releases/voatz-raises-22-million-seed-round-led-bymedici-ventures-300578763.html. [Accessed 14 August 2018].

[23] washingtonpost, "West Virginia to offer mobile blockchain voting app for overseas voters in November election," $\quad 2018 . \quad$ Anline]. https://www.washingtonpost.com/technology/2018/08/10/west-virginia-pilots-mobile-blockchainvoting-app-overseas-voters-november-election/?noredirect=on\&utm_term=.c7681a6672f2. [Accessed 15 August 2018].

[24] L. Horizon State Pty, "horizonstate," 2018. [Online]. Available: https://horizonstate.com/. [Accessed 15 April 2018]. 
[25] M. d. Castillo, "Russia Is Leading the Push for Blockchain Democracy," 2018. [Online]. Available: https://www.coindesk.com/russias-capital-leading-charge-blockchain-democracy/. [Accessed 23 August 2018].

[26] B. Mining, "Moscow's Blockchain Voting Platform Adds Service for High-Rise Neighbors," 2018. [Online]. Available: http://minebtc.co.uk/index.php/2018/03/15/moscows-blockchain-votingplatform-adds-service-for-high-rise-neighbors/. [Accessed 23 August 2018].

[27] coindesk, "Sumatra to Pilot Blockchain App for 'Decentralised Decision-Making'," 2018. [Online]. Available: https://www.coindesk.com/sumatra-to-pilot-blockchain-app-for-decentralised-decisionmaking/. [Accessed 15 August 2018].

[28] A. H. A. K. Ryan Henry, "Blockchain Access Privacy: Challenges and Directions," IEEE Security and Privacy, vol. 16, no. 4, pp. 38 - 45, 2018.

[29] D. D. L. K. Eric Piscini, "Blockchain \& Cyber Security. Let’s Discuss,” April 2017. [Online]. Available:

https://www2.deloitte.com/ie/en/pages/technology/articles/Blockchain_Cybersecurity.html\#. [Accessed 18 August 2018].

[30] E. F. M. C. Joanna Moubarak, "On blockchain security and relevant attacks," 2018 IEEE Middle East and North Africa Communications Conference (MENACOMM), pp. 1-6, 2018.

[31] X. J. M. Z. Wei Bi, "A Secure Multiple Elliptic Curves Digital Signature Algorithm for Blockchain,” 9 August 2018. [Online]. Available: https://arxiv.org/abs/1808.02988. [Accessed 19 August 2018].

[32] S. B. B. S. Uzun, "Bitter to Better - How to Make Bitcoin a Better Currency," Springer, vol. 7397, pp. 399-414, 2012. 